\title{
El alma en los diálogos Eudemo y el Protréptico y relación con el tratado Acerca del alma
}

\section{The soul in the dialogues Eudemus and Protrepticus, and relationship with the treatise On the Soul}

Joaquín García Alandete ${ }^{1}$

Resumen

Si bien Acerca del alma de Aristóteles puede ser considerado el primer tratado sistemático de una doctrina psicológica, le antecedieron el diálogo Eudemo y la exhortación a la filosofía Protréptico, que no han recibido tanta atención por parte de los estudiosos de la obra del Estagirita. En este trabajo se exponen las ideas centrales de estas dos obras en torno a la noción de alma, sus relaciones con el cuerpo y el intelecto humano, y su inmortalidad. Se contrastan estas ideas con algunos aspectos de la doctrina platónica y de la del tratado Acerca del alma, y se consideran interpretaciones contemporáneas.

Palabras clave:

Aristóteles, Eudemo, Protréptico, Acerca del alma, alma.

Abstract

Despite the Aristotle's On the soul can be considered the first systematic treaty of a psychological doctrine, it was preceded by the dialogue Eudemus and the exhortation to philosophy Protrepticus, which have not received as much attention from the researchers of the aristotelian phylosophy. This paper describes the main ideas of these two works on the concept of the soul and its relations with the body and the human intellect,and its immortality. These ideas are contrasted with some issues of the platonic doctrine and the treatise On the soul, and contemporary interpretations are considered.

Key words:

Aristotle, Eudemus, Protrepticus, On the soul, soul.

1 Universidad Católica de Valencia “San Vicente Mártir", Valencia, España. Correo electrónico: ximo.garcia@ucv.es 
Hay autores cuya obra no ha perdido un ápice de actualidad pese al tiempo transcurrido desde su redacción, sino que, por el contrario, sigue suscitando controversia y reflexión a través de las épocas, las escuelas y los autores (Blanco, 2002). Esto es lo que sucede con Aristóteles (384-322 a. C.), cuya originalidad, fecundidad, rigor y profundidad siguen asombrando y estimulando su estudio: "resulta sorprendente la influencia que el pensamiento de Aristóteles ha ejercido a lo largo de la historia. Cambiará sólo la intensidad o perspectiva, pero cada época ha tenido su Aristóteles"2. (Martínez Lorca, 1987, p. 5)

Es a lo largo del tiempo, a través de esa perenne actualidad, que la obra de Aristóteles, en su conjunto, ha sido interpretada de modos diversos. Así, durante siglos, desde la Antigüedad hasta el siglo $\mathrm{XX}$, se creyó que su pensamiento no había experimentado ningún tipo de cambio a lo largo de su vida, y se trató de explicar las incoherencias negando la autenticidad de alguna obra o forzando las interpretaciones para compatibilizar unas obras con otras. En la actualidad, por el contrario, se da por demostrado que no es así. Al respecto y como es sabido, Werner Jaeger distinguió tres periodos en el desarrollo de la obra aristotélica: (1) el de la Academia (367-347 a. C.), caracterizado por ser un periodo platonizante de la filosofía aristotélica; (2) el de los viajes (347-335 a. C.), un periodo de crisis interior, alejamiento del platonismo y desarrollo de la propia metafísica; y (3) el de madurez (335-322 a. C.), periodo de organización de la investigación, desarrollo del empirismo científico-natural (Jaeger, 1923/1994). Este esquema, como se sabe, sería revisado por Nuyens (1973). ${ }^{3}$

El caso es que, a pesar de su loable esfuerzo, la rigidez del esquema de Jaeger exigía forzar la interpretación de determinados pasajes de distintas obras para hacerlos coherentes con aquel. Por ejemplo, en relación con el estudio del alma, Jaeger estable-

2 La cursiva, como en el original.

3 Para una exposición genérica de las tesis de Jaeger, los estudios y críticas que suscitó, puede consultarse Alsina Clota (1986) y Lloyd (2007). ció que los capítulos 4 a 8 del libro III del tratado Acerca del alma ${ }^{4}$-que tratan sobre el noûs o intelecto- habrían sido redactados por Aristóteles en el primero de los tres periodos, mientras que los libros I y II pertenecerían al tercer y último periodo, marcados por la evolución de su pensamiento específico y su progresivo alejamiento -incluso contraposición- de la filosofía platónica.

Sin dejar de reconocer los méritos de Jaeger, otros autores rechazan algunas de sus conclusiones y consideran que ciertos aspectos de su cronología deben corregirse (p. ej., Lloyd, 2007, pp. 31-37). Ross (1981, p. 27) afirma que el libro III de Acerca del alma pertenece a la época de residencia de Aristóteles en Tracia, Lesbos y Macedonia y, por su parte, Mansion (1954) corrige algunas afirmaciones de Jaeger sobre los libros de la Metafísica y muestra que la continuidad del pensamiento aristotélico a lo largo de sus obras es más importante que las diferencias. A ello hay que añadir que, según recientes investigaciones, la génesis y composición de los tratados didácticos de Aristóteles son más complejos de lo que Jaeger supuso. En cuanto a la tesis del progresivo alejamiento y definitivo abandono del platonismo, parece no ser cierto, al menos en relación con algunos aspectos de la filosofía aristotélica. Así, en lo que respecta a la concepción del alma, en Acerca del alma admite Aristóteles que una parte de la misma -el intelecto activo, noûs poietikós- es inmortal. Por lo que hace a Acerca del Alma en su conjunto, en particular, existen razones suficientes para afirmar con rotundidad que es un tratado unitario y coherente de principio a fin redactado en un solo momento (Berti, 1962; Calvo Martínez, 2003a, especialmente pp. 120-124; De Vogel, 1960, pp. 248256; Elordue, 1950; Gigon, 1960; Nuyens, 1973).

En relación con el objetivo de este trabajo, Nuyens propuso, para una comprensión adecuada de la doctrina aristotélica del alma, partir no de Acerca del alma -que sería el terminus ad quem-,

4 En el presente trabajo se ha utilizado la edición de Bekker (1831) para la numeración de las citas textuales y la edición de Calvo Martínez (Aristóteles, 2003) para el texto en español. 
sino del Eudemo (Nuyens, 1973)5. Por su parte, Lloyd afirma que un análisis de Eudemo y de Protréptico permite responder a cuestiones tales como si hubo en la vida de Aristóteles un periodo de platonismo ortodoxo, si mantuvo la doctrina de las ideas o del alma, o si ya en sus obras iniciales se mostró contrario a Platón (Lloyd, 2007, pp. 31-45; Vallejo Campos, 2005a). Teniendo en cuenta esto, dedicamos este trabajo a la exposición de las ideas sobre el alma en los diálogos Eudemo y Protréptico, puesto que en ellos Aristóteles expone aspectos de su doctrina sobre el alma, su relación con el cuerpo y el intelecto que bien se mantendrán con matices, bien serán sustituidos por otros en Acerca del alma.

\section{Del alma y del intelecto en el Eudemo ${ }^{6}$}

Titulado así en recuerdo de un amigo y condiscípulo -Eudemo de Chipre, muerto en 354 a.C.--, el diálogo Eudemo es una obra juvenil de Aristóteles, del periodo en el que fue miembro de la Academia platónica (367-347 a. C.), de la que solo se conservan seis fragmentos auténticos (Ross, 1981) y que tienen su paralelo en Acerca del alma y en Tratados breves de historia natural. El hecho de estar motivada por la muerte del amigo y condiscípulo explica "no pocas características de la obra, como su ascetismo y su pesimismo en la concepción de la vida terrena o su insistencia en la inmortalidad y la felicidad del alma después de la muerte" (Vallejo Campos, 2005b, p. 96). Características que, junto a las ideas acerca de la preexistencia del alma y la supervivencia de las experiencias mundanas que ésta tuvo durante la existencia terrena, hacen de este diálogo un texto de, para algunos, clara adhesión a la doctrina platónica $\mathrm{y}$ contraste con su pensamiento de madurez.

5 Cfr. Elorduy (1950).

6 Se ha usado la edición de Vallejo Campos de los Fragmentos de Aristóteles para Gredos.

7 Rose (1967), como es sabido, consideró espúreos los diálogos aristotélicos (tesis sostenida en Aristoteles pseudepigraphus, Leipzig, 1863), por no ser coherentes con los escritos del Corpus Aristotelicum. Zürcher (1952) afirmó que solo entre el 20 y el 30 por ciento del Corpus era realmente obra de Aristóteles, atribuyendo el resto a Teofrasto; asimismo, que Aristóteles había permanecido platónico durante toda su vida.
Al igual que Protréptico y otras obras, Eudemo forma parte de las obras aristotélicas consideradas exotéricas (lógoi exoterikoi o akroamatikoi), es decir, aquellas que estaban dirigidas al lector externo a la Academia, y su interpretación se hace "harto difícil, por no decir enigmática” (Martínez Lorca, 1987, p. 11). En Ética a Nicómaco (1102a18-28), Aristóteles se refiere a Eudemo al afirmar que en una de sus obras exotéricas se habla del alma, cuya investigación es de indudable interés para quien pretenda conocer la sociedad humana.

Aristóteles reflexiona sobre el alma -su inmortalidad, si es la de toda ella o solo la de la racional o noûs, sobre la actividad del noûs como independiente del cuerpo-, entre otros asuntos tales como la veracidad de los sueños y la anámnesis. Como se ha señalado, acepta en esta obra las doctrinas platónicas sobre la preexistencia, la transmigración y la reminiscencia, y critica el concepto de alma como armonía, al igual que hiciera Platón en Fedón (Ross, 1981) ${ }^{8}$. Temáticas estas últimas que encontramos también en el diálogo Sobre la filosofía -que Nuyens (1973) data en la misma etapa que Protréptico, es decir, unos años después que Eudemo- y que tendrán continuidad en Acerca del alma.

Según Jaeger (1947), Eudemo manifiesta la dependencia de la metafísica platónica, tanto en el rechazo del materialismo como en otras cuestiones positivas, a la vez que la independencia de la lógica y la metodología platónicas, concluyendo que aquélla y ésta mantenían entre sí una débil conexión en la mente de Aristóteles. A pesar del intento de Jaeger de fundamentar estas ideas, resultan inconsistentes a la luz de ciertas cuestiones -como la del carácter sustancial del alma, expuesta en el fragmento 54que le llevan a forzar una "hermenéutica" de Eudemo poco sostenible (Martínez Lorca, 1987, pp. 11-13). Precisamente, el hecho de que, como se ha señalado, algunos de los temas -centrales, por otra partetratados en el Eudemo son retomados en Acerca del alma, ofrece más fundamento a la tesis de que en el

8 La psicología expuesta en Eudemo es exclusivamente humana, mientras que la de Acerca del alma es una psicología general, relativa a cualquier ser vivo, sea vegetal, animal o humano. 
Universidad Pedagógica Nacional

Facultad de Humanidades

pensamiento de Aristóteles existe continuidad entre un periodo y otro (Berti, 1962; Lloyd, 2007).

Los fragmentos 2, 3 y 5 hacen clara alusión a las doctrinas sobre la inmortalidad del alma, cuando menos del intelecto; y la reminiscencia. Al respecto, en el fragmento 5 se afirma que "el alma, al llegar aquí procedente del más allá, olvida las visiones contempladas en aquel lugar, mientras que cuando se marcha de aquí recuerda allá sus experiencias de este mundo" y que "para las almas, la vida sin el cuerpo, al estar en concordancia con la naturaleza, se asemeja a la salud, mientras que su vida en los cuerpos, al ser contraria a la naturaleza, se asemeja a la enfermedad". Estas ideas evidencian la consideración negativa que merecen el cuerpo y la vida del alma en el mismo, en consonancia con la doctrina platónica del cuerpo como cárcel del alma y el anhelo de esta por escapar de aquél y retornar a la existencia extracorpórea. La existencia misma es una pena de la que hay que escapar, puesto que no ha sido evitable. El fragmento 6 contiene esta misma idea, expresada en términos extremos:

\begin{abstract}
Muchos hombres sabios [...] se han lamentado de la condición humana, considerando que la vida es un castigo y que el nacimiento del hombre es, por principio, la mayor desgracia. [...] no haber nacido es lo mejor de todo y que haber muerto es mejor que vivir. [...] lo primero entre las cosas que están al alcance de los hombres: una vez nacidos, morir tan pronto como sea posible. [...] la existencia en la muerte es mejor que la que tenemos en vida.
\end{abstract}

El alma es una sustancia simple cuyas acciones se expresan a través del cuerpo (fragmento 11b), distinta del cuerpo, siendo accidental la unión entre ambos. Según Nuyens (1973) la concepción de la relación entre alma y cuerpo en Eudemo es propia de la primera etapa del desarrollo filosófico aristotélico: alma y cuerpo son sustancias distintas -incluso opuestas-, cuya unión es accidental -incluso violenta- $y$ temporal. El alma es preexistente al cuerpo $y$, tras la muerte del cuerpo, retorna a su estado anterior. Entonces, mientras que el cuerpo es perecedero, el alma es inmortal (Aristóteles, trad. en 2005, pp.
96-117). Por tanto, alma y cuerpo son separables (fragmento 11a). Es, claramente, un elemento de la concepción platónica del alma (Berti, 1962).

Por otra parte, Aristóteles, al igual que ya hiciera Platón en Fedón, se muestra contrario a la doctrina de la armonía del alma, siendo esta ontológicamente anterior y superior al cuerpo (fragmentos 7a, 7d, 7e y 7f). Siguiendo a Jaeger (1923/1994), estas ideas manifiestan la dependencia aristotélica de la metafísica platónica, en cuanto al rechazo del materialismo y en cuanto a la aceptación de la inmortalidad del alma. El rechazo de la idea del alma como armonía aparecerá de nuevo en Acerca del alma, haciendo evidente la continuidad de ciertas ideas sobre la concepción del alma a través de las distintas etapas en la evolución del pensamiento aristotélico. Para Aristóteles, el alma es esencialmente una realidad comprensible como separable del cuerpo.

En cuanto a la actividad del alma racional, el fragmento 8 señala que el alma racional media entre lo divisible y lo indivisible, siendo una cierta forma ella misma y receptora de las formas -si bien en potencia, pues las formas verdaderas corresponden al intelecto, que es superior al alma-. Al respecto, Guthrie (1999) interpreta el texto de Simplicio en el que este afirma que, según Aristóteles, "el alma es receptora de las formas, no en su totalidad sino el alma intelectiva, dado que conoce las formas que son verdaderas secundariamente, pues las formas verdaderas corresponden al intelecto, que es superior al alma", en el sentido de que son conocidas en potencia, al modo en que ello es entendido en Acerca del Alma. Este fragmento, entre otros, es para Guthrie comprensible solo considerando globalmente la psicología aristotélica y, especialmente, la doctrina de la materia y la forma. Este autor, asimismo, subraya que en Acerca del alma (III 4, 412a19-21), Aristóteles denomina al alma "forma", si bien ya no como esencias separadas de las cosas empíricas al modo de la filosofía platónica. Con todo, hay que distinguir claramente entre la concepción del alma en Eudemo y en Acerca del alma, como ya así sugieren los fragmentos 3 y 4 . 
Por otra parte, la función más elevada y noble (kyriótaton ergón) del alma es la inteligencia, orientada naturalmente al saber y cuyo objeto supremo es la verdad. Ese fin que es el conocimiento orientado por la sabiduría (phrónesis) tiene su punto de realización máxima en el conocimiento contemplativo (theorikèn epistemen) de la verdad, actividad que supone la virtud -vida conforme a lo mejor según la propia naturaleza- $y$, por ende, la felicidad:

En la medida en que aquellos que han sobrepasado estos objetos de la opinión, de carácter mixto y multiforme, se elevan por medio del pensamiento hasta aquel objeto primero, simple e inmaterial $y$, al aprehender realmente su verdad más pura, creen que han alcanzado la culminación de la filosofía, como en un misterio. (fragmento 10)

\section{Del alma y del intelecto en el Protréptico ${ }^{9}$}

Diálogo de juventud, al igual que Eudemo, es concretamente una exhortación a la filosofía. $Y$, al igual que Eudemo, ha suscitado el debate sobre su adhesión o, por el contrario, autonomía con respecto a las doctrinas platónicas sobre tales cuestiones (Berti, 2008; Düring, 1961; González Escudero, 2010; Lloyd, 2007, pp. 40-45; Seggiaro, 2011; Vallejo Campos, 2005c, pp. 125-129). En este diálogo, del que se conservan solo fragmentos, Aristóteles reflexiona sobre la filosofía, exhortando a su cultivo. Como se sabe, esta obra sería de gran influencia posterior, así en Cicerón (Hortensius) y, a través de éste, en San Agustín de Hipona (Confesiones).

La actividad filosófica, autotélica y propiciadora de la felicidad -o es ella misma (fragmento 6 B68)-, más que cualquier posesión del cuerpo u otros bienes materiales (p. ej., fragmentos 3, 4, 5 y 6, 9 B99), consistiría en el cultivo de la sabiduría, en el sentido de phrónesis ${ }^{10}$, máxima virtud noética, mediante el conocimiento de los principios prime-

9 Ver nota 4.

10 Vallejo Campos (2005c, p. 133) señala que en Protréptico la phrónesis "parece designar la inteligencia como se cultiva en el ámbito del saber filosófico y lo característico es que en ella se aúnan lo teórico y lo práctico. [...] no tiene como objetivo la producción de algo externo y diferente de ella misma, sino que constituye una facultad cuyo ejercicio es un fin en sí mismo". ros de lo real. Aristóteles exhorta al hombre en esta obra, en definitiva, a "abocarse al conocimiento de los principios que rigen lo real, pues sólo por medio de este conocimiento logrará plenificar su naturaleza $y$, por ende, alcanzará la verdadera felicidad. Ahora bien, según Aristóteles, para alcanzar dicho conocimiento el hombre debe ejercitarse en la phrónesis (fragmento 35) y, por este motivo, debe filosofar". (Seggiaro, 2011, p. 420)

La identificación del hombre con, especialmente, la parte racional del alma (fragmentos 17 y 23), el movimiento concebido como proceso de búsqueda de los primeros principios y la concepción teleológica de la naturaleza son presupuestos de la distinción aristotélica entre ciencias teóricoprácticas y productivas (fragmento 9): mientras que las primeras tienen como objetivo el conocimiento de los principios de lo real, lo bueno y la verdad (fragmentos 9,42 y 65 ) y se relacionan con la parte racional del alma; las segundas tienen como objetivo la satisfacción de las necesidades biológicas y el cuidado del cuerpo. (Berti, 2008; Düring, 1961; Seggiaro, 2012; Vallejo Campos, 2005c)

La actividad filosófica es propia del alma, concretamente de la parte racional, que es la mejor, lo que en cierto modo somos y que de manera especial gobierna y utiliza. El cuerpo mantiene con el alma una relación de subordinación, es gobernado y usado, a modo de instrumento o herramienta idea que aparecerá también, por ejemplo, en Ética a Eudemo VII 9, 1241b17-24 y en Política I 5, 1254a34$36^{11}-$, a pesar de que considera que el cuerpo es constitutivo de la naturaleza humana (fragmentos 6, B23 y B26)

La naturaleza en su totalidad, como si estuviera dotada de razón, no hace nada al azar, sino todo con vistas a algo, y, desterrando el azar, la naturaleza atiende al fin más incluso que las artes, porque también las artes eran imitaciones de la

11 Ética a Eudemo se sitúa cronológicamente entre Protrépticoy Ética a Nicómaco, es decir, en la etapa de transición del desarrollo de la filosofía aristotélica, según Jaeger y Nuyens. Por su parte, si bien Ios capítulos I, IV, V y VI de Política podrían pertenecer al último periodo aristotélico, según Jaeger, serían anteriores en todo caso a Acerca del alma, según Nuyens. 
naturaleza. Al estar constituido el hombre por naturaleza de alma y cuerpo, siendo el alma mejor que el cuerpo y ordenándose siempre lo peor para servir con vistas a lo mejor, también el cuerpo existe con vistas al alma. Ahora bien, en el alma una parte estaba dotada de razón y otra carecía de ella, que era precisamente la peor, de manera que la parte irracional se ordena con vistas a la que está dotada de razón. Ahora bien, el intelecto radica en la que está dotada de razón, de forma que la demostración nos obliga a afirmar que todo [en el hombre] existe con vistas al intelecto. (fragmento B23)

El alma es, pues, superior al cuerpo; de hecho, este es un castigo (fragmento 10b1 B107), recordando esta idea a la de Platón en Fedón, como lo es la vida en su conjunto, anhelando el alma volver a "la isla de los bienaventurados" (fragmento 12a), de donde ha venido (fragmento 15 B96); y en ella, en el alma, se pueden distinguir dos partes: racional e irracional. Esta idea -entre algunas más similares a las contenidas en Protréptico- aparece en otras obras, como en Tópicos (V 1, 128b14-19), en la que Aristóteles afirma que el alma es capaz de dominar y el cuerpo de ser dominado, en Física (IV 5, 212b11-129), donde afirma que el alma "está accidentalmente en un lugar", que no es otro que el cuerpo, y en Sobre la generación y la corrupción (II 7, 334a9-15), donde afirma que "ninguna afección del alma es corpórea”.

Las actividades del alma son preferibles a las del cuerpo -productivas-, y de entre aquellas, las racionales -teóricas y prácticas- son preferibles a las irracionales -meramente fisiológicas-, relativas a la supervivencia. Aristóteles establece en este diálogo, pues, una distinción ontológica entre cuerpo y alma que, pese a su concepción del hombre como un compuesto, implican una cierta dosis de platonismo, al menos moderado, a juicio de algunos intérpretes (p. ej., Nuyens, 1973). Moderado en todo caso, pues reconoce que entre cuerpo y alma se da una relación de colaboración en la que la contribución de aquel es necesaria, viniendo a constituir elemento imprescindible para la actividad superior del alma.
El hombre, en virtud de que lo superior es preferible a lo inferior, tendrá la máxima realización de su auténtica naturaleza en el cultivo de la actividad de la parte racional del alma. Y en ella reside el noûs, que es la facultad racional superior y que iguala al hombre a la divinidad: "privado de percepción e intelecto, el hombre se hace semejante a una planta, pero si está privado sólo de intelecto se animaliza, en cambio, cuando está libre de irracionalidad y persevera en su intelecto se asemeja a dios". (fragmento B28)

La verdad es la función propia de la parte racional del alma (fragmento 6 B65). Y el cultivo de la actividad del noûs - la sabiduría o contemplaciónsupone la felicidad del hombre, alcanzar el anhelo máximo de su existencia y la forma de vida más apropiada a su naturaleza (fragmentos 14 y 15).

En el fragmento 14 B80, Aristóteles introduce la distinción entre acto y potencia en relación con la vida y el intelecto, cuestiones esenciales cuando en Acerca del alma conciba el alma como acto primero de un cuerpo que en potencia tiene vida y distinga entre intelecto activo e intelecto pasivo. Y, utilizando una comparación que reaparecerá en Acerca del alma, remite a la visión como acto y como potencia:

Vivir parece decirse de dos maneras, en un sentido según la potencia y en otro según el acto, pues decimos que son videntes todos aquellos animales que tienen vista y están capacitados por naturaleza para ver, aunque suceda que tengan los ojos cerrados, e igualmente aquellos que están utilizando esta facultad y ejercitando la visión. Lo mismo ocurre con saber y conocer, unas veces lo decimos en el sentido de hacer uso de esta facultad y estar pensando, otras veces, en el sentido de estar en posesión de ella y tener la ciencia. (fragmento 14 B80)

Será el ejercicio o actividad del intelecto, y no la mera posesión de su posibilidad, lo que en mayor medida será apropiado al mismo (fragmentos 14 B81ss.). Ahora bien, la actividad del intelecto no es la contemplación de las ideas platónicas, sino que su intelección consiste en "visiones de objetos inteligibles de la misma manera que es actividad de la 
vista la visión de los objetos sensibles" (fragmento B24). Idea obviamente central en la doctrina aristotélica del intelecto -qué es lo que intelige el intelecto y cómo lo intelige - y que también reaparecerá en Acerca del alma. En los fragmentos 48 y 75-77, Aristóteles distingue y jerarquiza los razonamientos de primer y segundo orden -los que parten de los primeros principios y los que parten de los datos de la sensibilidad, respectivamente- $y$ las distintas ciencias -siendo la filosofía la que tiene como objeto los objetos más nobles-. Esta distinción entre tipos de razonamiento y de conocimiento la expresará posteriormente en Acerca del alma como sigue. El entendimiento, por una parte, se halla en potencia para el conocimiento de las cosas, recibiendo la forma de sus semejanzas y, en tal sentido, es pasivo (Nuyens, 1973). Por otra parte, los inteligibles son en potencia, no en acto -a diferencia de las ideas, que Platón entendía en acto-. Entonces, la actividad del intelecto consistirá en elaborar sus objetos de conocimiento, actuando sobre las imágenes facilitadas por la sensibilidad. Aristóteles, pues, desarrolla una doctrina sobre el intelecto que se encuentra, equilibradamente, entre las teorías materialistas-Demócrito-e idealistas -Platón- del conocimiento. Si bien el conocimiento empieza con la sensibilidad, no se agota en la misma, existiendo entre esta y el intelecto una línea de continuidad. (Cfr. Berti, 2005, especialmente p. 55; Zucca, 2006, especialmente p. 279)

Con todo, a juicio de Benavente Barreda (1968), Aristóteles no ofreció nunca una argumentación del todo satisfactoria acerca de cómo se produce el paso de los datos físicos, captados por la sensibilidad, a la forma o esencia, inteligida por el intelecto:

El "cómo" exacto del paso de esta información física a la información intencional es un hiato que Aristóteles no aclaró [...]. Pero Aristóteles entiende que entre la acción de la forma física y la potencia -que al fin y al cabo es potencia del órgano- existe una cierta connaturalidad, y ésta permite la información. Sin embargo aquí, al hablar del entendimiento agente, niega de modo taxativo que éste sea influible de ningún modo el fantasma, así considerado, no puede ser interpre- tado en ningún momento como causa eficiente que influya en el noûs, de modo que éste llegue a la esencia. El fantasma, la imagen, ejercerá, todo lo más, una función de causa material: será de donde el noûs -que es activo- tomaría sus materiales para hacer con ellos la idea. (Benavente Barreda, 1968, p. 63)

Por último, la doctrina sobre la inmortalidad del alma que Aristóteles expone en Protréptico hay que considerarla, al igual que la de Eudemo, desde la concepción de las relaciones entre cuerpo y alma (Nuyens, 1973, p. 239). Pues bien, la idea de la inmortalidad del intelecto la expresaba Aristóteles en Protréptico, afirmando que "nada divino o bienaventurado pertenece a los hombres, a excepción de aquella única cosa digna de consideración, que es cuanto hay en nosotros de inteligencia y sabiduría. Pues, sólo esto parece ser inmortal en nosotros y sólo esto es divino" (fragmento 10c1). En Acerca del alma, Aristóteles nos dice que el intelecto activo es separable, simple -sin mezcla- e impasible, puesto que es esencialmente acto, y superior al pasivo -"lo inteligible ha de estar en él del mismo modo que en una tablilla en la que nada está actualmente escrito: esto es lo que sucede con el intelecto" (Acerca del Alma III 4, 429b31-430a1)-. Asimismo, afirma que "la misma cosa son la ciencia en acto y su objeto" (Acerca del alma III 5, 430a19-20) y, además:

Desde el punto de vista de cada individuo la ciencia en potencia es anterior en cuanto al tiempo, peor desde el punto de vista del universo en general no es anterior ni siquiera en cuanto al tiempo: no ocurre, desde luego, que el intelecto intelija a veces y a veces deje de inteligir. (Acerca del alma III 5, 430a20-22)

Asimismo, afirma:

Una vez separado es sólo aquello que en realidad es y únicamente esto es inmortal y eterno. Nosotros, sin embargo, no somos capaces de recordarlo, porque tal principio es impasible, mientras que el intelecto pasivo es corruptible y sin él nada intelige. (Acerca del Alma III 5, 430a22-25)

Así pues, si bien redactados en épocas distintas y desde unas claves intelectuales diferentes, 
Universidad Pedagógica Nacional

Facultad de Humanidades

Protréptico y Acerca del Alma coinciden en lo esencial, mostrando que en ciertos aspectos clave la doctrina de Aristóteles sigue un hilo conductor, responde a unos tópicos que se mantienen desde sus años de juventud a su madurez.

\section{Conclusión}

Entre Eudemo y Protréptico, redactados durante el primer periodo de la filosofía aristotélica (367-347 a. C.), y Acerca del alma, redactado durante el tercer y último periodo (335-322 a. C.), hay cambios importantes en la doctrina aristotélica del alma, si bien en una línea de continuidad progresiva y coherente, desde planteamientos platonizantes a planteamientos autónomos. El eje sobre el que gravitan tales cambios es la concepción de las relaciones entre cuerpo y alma. Siguiendo a Nuyens (1973), cuerpo y alma forman para Aristóteles, en su primer periodo, una unidad meramente accidental y temporal, por tanto no necesaria, coincidiendo en esto con Platón; más todavía, entre cuerpo y alma existe una diferencia y abismo ontológico, son separables y el cuerpo no solo es inferior al alma, sino que es su cárcel. Durante su segundo periodo, Aristóteles considera que la relación entre cuerpo y alma es instrumental, de manera que el alma -dominante, superior- utiliza el cuerpo -dominado, inferior- para ejecutar sus intenciones. Para el último Aristóteles, el de Acerca del alma, el cuerpo y el alma constituyen en el ser vivo una unidad sustancial: no pueden darse sino de tal manera, puesto que el alma es la forma del cuerpo, su entelequia, aquello por lo que este es lo que es y no otra cosa, aquello por lo que el cuerpo tiene su naturaleza propia, específica, su actualidad. El ser vivo es un compuesto de forma y materia, inseparablemente, un todo complejo y único -teoría hilemórfica-. Los ejemplos del hacha (Acerca del alma II 1, 412b11ss.) y del ojo (Acerca del alma II 1, 412b18ss.) expresan con claridad el tipo de relación entre forma y materia -entre alma y cuerpo por tanto, en el caso de los seres vivos-; la unidad sustancial entre materia y forma está por encima de la heterogeneidad de las partes, siendo explicada en virtud de la forma como actualidad primera de la materia. Por otra parte y en virtud de esto mismo, las diferentes potencias, facultades u operaciones del organismo se atribuyen al sujeto sustancial en su unidad y totalidad, y no al cuerpo o al alma por separado, como es el caso de Eudemo y Protréptico. La preexistencia y la transmigración del alma, así como su oposición ontológica con respecto al cuerpo, ideas propias del primer Aristóteles, el de la Academia, han sido desterradas en la doctrina psicobiológica del último Aristóteles, el del Liceo: siendo principios diferentes, alma y cuerpo contribuyen en su unión a dar lugar a un solo ser natural y sustancial.

\section{Referencias}

Alsina Clota, J. (1986). Aristóteles. De la filosofía a la ciencia. Barcelona: Montesinos.

Aristóteles (Trad. en 2003). Acerca del alma [introducción, traducción y notas de Tomás Calvo Martínez]. Madrid: Gredos.

Aristóteles (Trad. en 2005). Eudemo o Acerca del Alma. En Aristóteles, Fragmentos (pp. 96-117) [introducción, traducción y notas de Álvaro Vallejo Campos]. Madrid: Gredos.

Bekker, I. (1831). Aristoteles Opera, Vol. I. Berlín: Academia Regia Borussica. Recuperado de http://ia700500. us.archive.org/7/items/aristotelisopera01arisrich/ aristotelisopera01arisrich_bw.pdf

Benavente Barreda, J. M. (1968). El problema del concepto abstracto en Aristóteles. Logos: Anales del Seminario de Metafísica, 3, 51-74.

Berti, E. (1962). La filosofía del primo Aristotele. Padua: Cedam.

Berti, E. (2005). Nuovi studi aristotelici. Brescia: Morcelliana.

Berti, E. (2008). Aristotele Protreptico: Esortazione alla filosofía. Turín: UTET.

Blanco, C. (2002). Aristóteles, un pensamiento de actualidad. Ensayos: Revista de la Facultad de Educación de Albacete, 17, 11-48.

Calvo Martínez, T. (2003a). Introducción. En Aristóteles, Acerca del alma (pp. 97-130). Madrid: Gredos.

De Vogel, C. J. (1960). The legend of Platonizing Aristotle. En I. Düring y G. E. L. Owen (Eds.), Aristotle and Plato in the Med-Fourth Century. Papers of the 
Symposium Aristotelicum held at Oxford in August, 1957 (pp. 248-256). Götteborg: Almquist and Wiksell.

Düring, I. (1961). Aristotle's Protrepticus. An Attempt at Reconstruction. Götteborg: Almqvist and Wiksell.

Elorduy, E. (1950). La evolución de la psicología de Aristóteles según Nuyens. Pensamiento: Revista de Investigación e Información Filosófica, 6, 465-493.

Gigon, O. (1960). Prolegomena to an edition of the Eudemus. En I. Düring y G. E. L. Owen (Eds.), Aristotle and Plato in the Mid-Fourth Century. Papers of the Symposium Aristotelicum held at Oxford in August, 1957 (pp. 20-30). Götteborg: Almquist and Wiksell.

González Escudero, S. (2010). Protréptico. Aristóteles. Eikasia. Revista de filosofía, 30, 1-22.

Guthrie, W. K. C. (1999). Historia de la filosofía griega. Vol. VI: Introducción a Aristóteles. Madrid: Gredos.

Jaeger, W. (1923/1994). Aristóteles. Bases para la historia de su desarrollo intelectual. México: Fondo de Cultura Económica.

Lloyd, G. (2007). Aristóteles. Buenos Aires: Prometeo.

Mansion, A. (1954). Het Aristotelisme in het historische Perspectief. Platonisme, Aristotelisme, Neoplatonisme. Bruselas: Paleis der Academiën.

Martínez Lorca, A. (1987). Prólogo. En Averroes, Comentario al libro De Anima de Aristóteles (pp. 5-84). Madrid: Universidad Nacional de Educación a Distancia.

Nuyens, F. (1973). Lévolution de la Psichologie d'Aristote. Lovaina : Éditions de l'Institut Supérieur de Philosophie.

Rose, V. (1967). Aristotelis qui ferebantur librorum fragmenta, collegit $V$. Stuttgart: Teubner.

Ross, W. D. (1981). Aristóteles. Buenos Aires: Charcas.

Seggiaro, C. (2011). Resonancias platónicas en la serie de fragmentos 33-36 del Protréptico de Aristóteles. Areté. Revista de filosofía, 23(2), 399-420.

Seggiaro, C. (2012). La relación alma y cuerpo en el Protréptico de Aristóteles: hacia una jerarquización de las ciencias. Sapere Aude, 3(5), 2-26.

Vallejo Campos, A. (2005a). Introducción. En Aristóteles, Fragmentos (pp. 7-23). Madrid: Gredos.

Vallejo Campos, A. (2005b). Introducción. En Aristóteles, Fragmentos (pp. 95-98). Madrid: Gredos.

Vallejo Campos, A. (2005c). Introducción. En Aristóteles, Fragmentos (pp. 125-137). Madrid: Gredos.
Zucca, D. (2006). Essere, linguaggio, discorso. Aristotele filosofo dell’ordinario. Milán: Mimesis.

Zürcher, J. (1952). Aristoteles' Werk und Geist. Paderborn: F. Schöningh. 